\title{
Combining The Tunneling And Anomaly Phenomena In Deriving the Gravitational Anomaly
}

\author{
Subir Ghosh 1 \\ Physics and Applied Mathematics Unit, Indian Statistical Institute \\ 203 B.T.Road, Kolkata 700108, India
}

\begin{abstract}
:
In the present work we have derived the gravitational anomaly from a fundamentally different perspective: it emerges due to the tunneling of particles (in the present case fermions) across the black hole horizon. The latter effect is in fact the Hawking radiation. We have used the analogy of an early idea [17, 18] of visualizing chiral gauge anomaly as an effect of spectral flow of the energy levels, from the negative energy Dirac sea, across zero energy level in presence of gauge interactions. This was extended to conformal anomaly in [23]. In the present work, we exploit the latter formalism in black hole physics where we interpret crossing the horizon of black hole (the zero energy level) as a spectral flow since it is also accompanied by a change of sign in the energy of the particle. Furthermore, Hawking radiation induces a shrinking of the radius of the horizon [15, 16] which reminds us of a similar rearrangement in the Fermi level generated by the spectral flow [17, 18, 21]. Hence in our formulation the negative energy states below horizon play a similar role as the Dirac sea. We successfully recover the gravitational anomaly.
\end{abstract}

Among the various approaches to derive Hawking radiation [1], two have recently stood apart in popularity: (i) the tunnelling mechanism by Parikh and Wilczek [2] and (ii) anomaly mechanism by Robinson and Wilczek [3]. Furthermore, there have been numerous refinements in the computational procedure [4]-[14]. Quite surprisingly, it seems that these two

\footnotetext{
${ }^{1}$ E-mail: sghosh@isical.ac.in
} 
mechanisms are disconnected both in conceptual as well as computational sense. Indeed, it would be very satisfying if this situation can be improved. In the present Letter we propose a novel conjecture that the two phenomena are complimentary to each other: the tunnelling across the horizon (or the Hawking radiation) generates the gravitational anomaly. This is quite in keeping with the spirit of [15, 16] where it is shown that crossing of the horizon actually creates the potential barrier through which the particle tunnels. We interpret the crossing of the horizon by a particle as a form of spectral flow across the zero energy level that is the particle at the horizon, that preferentially creates one type of chirality thereby generating the anomaly. The experts in anomalies will immediately notice that we have simply borrowed the intuitive and physical mechanism [17, 18, 19, 20, 21, 22, 23, 24] of the generation of chiral gauge anomaly [25, 26] where the $U(1)$ gauge interaction induced a spectral flow across the zero energy state or the Fermi level in Condensed Matter Physics [21].

One immediately notices a striking conceptual similarity between the two phenomena: spectral flow across the Fermi level in case of gauge anomaly [17, 18, 21] and particle crossing the horizon in case of black hole, especially along the lines of Parikh [15, 16]. It has been emphasized by Nielsen and Ninomiya [17, 18] that the Fermi level gets rearranged or shifted due to the spectral flow so that states of one chirality are preferentially produced. In the language of Parikh [15, 16] also, due to Hawking radiation (or equivalently negative energy particle falling in the black hole) the size of the event horizon size of the black hole gets reduced, in order to maintain energy conservation. Furthermore, in both the instances of chiral anomaly and gravitational (or diffeomorphism) anomaly, one gets identical contributions coming from particle and anti-particle channels that eventually add up to yield the final anomaly expression. The theory becomes chiral because the ingoing modes can not influence the physics outside the horizon and hence drops out from contention. Indeed, this indicates that the Fermi level in Condensed Matter Physics and event horizon in black hole physics play analogous roles in the present instance. 
Two ideas stand out in the derivation of Hawking radiation via tunnelling [2]: firstly the signature of energy changes as a particle crosses the Schwarzschild radius or horizon (that is induced by the metric) and secondly the Hawking radiation survives because one partner of a particle-antiparticle pair crosses the great divide (horizon) and essentially becomes lost as far as the outside world is concerned. The final Hawking radiation gets equal contribution from both particle and antiparticle sectors.

Let us now recall the spectral flow mechanism [17, 18, 19, 20, 21, 22, 23] (see also [24] for the simplest example) for the chiral anomaly [25, 26], induced by the negative energy Dirac sea for fermions. Even though the Dirac sea has lost some of its ground in Quantum Field Theory, it is probably the only option that one has in understanding the anomaly phenomenon in an intuitive way and it should be stressed that the Dirac sea approach [17, 18, 23. reproduces correctly the numerical factors in different types of anomaly expressions as well. The main idea here is that although classically the two chiralities of a massless fermion (say an electron in the massless limit) interacting with electromagnetic field are completely decoupled, they actually become entangled through quantum effects in the presence of Dirac sea. The gauge field induces a (spectral) flow of the states across the zero energy level for massless particle. This preferentially creates one type of chirality, (.e.g. right-handed antiparticles in the Dirac sea and left-handed particles), and gives rise to the chiral anomaly, or non-conservation of chiral charge, even though overall charge conservation is maintained. Exactly similar circumstance also prevails when one considers chiral fermions in curved background. It was shown by Fumita [23] that one obtains the gravitational anomaly. In fact quantum mechanical analysis is enough for the gauge anomaly but quantum field theory is needed for the gravitational anomaly [23].

It has been emphasized [22] that the whole of the infinite negative energy Dirac sea contributes to the anomaly as that constitutes the ground state of the system. But this is not apparent in the derivation of the gauge anomaly via this scheme [17, 18, 24] where the correct expression for the chiral anomaly, including numerical factors, is reproduced by 
considering the contribution of the zero (or Fermi energy) level only. This puzzle was solved (in a somewhat unnoticed work) by Fumita [23] who, to our knowledge for the first time, considered the conformal anomaly also in the spectral flow framework. Fumita introduced a regularization scheme - an exponential cutoff $(\Lambda)$ for large energy - to tackle the infinite negative energy sea and obtained both the chiral and conformal anomaly by integrating over the Dirac sea. Now it becomes clear in this unified study that this formal procedure is really redundant for the gauge anomaly where one is concerned with the fermion number current only and the energy of the levels does not explicitly come into play. In all the intermediate states between energies zero and $\Lambda$ the spectral flow just shifts the particles from one level to another (recall the argument of putting guests in a full hotel having an infinite number of rooms in [22]) and the overall non-trivial effect can be obtained by considering the spectral flow effect at only the zero energy level [17, 18, 24] or at only the bottom level $\Lambda$ [23]. On the other, when one considers the gravitational phenomena, the energy-momentum current appears and to get the ground state energy one needs to integrate the energy of an arbitrary level over the whole (properly regularized) Dirac sea and the whole Dirac sea explicitly contributes 23 .

Finally we come to our conjecture that both of the above two phenomena, Hawking radiation in the tunnelling mechanism [2] and gauge anomaly induced by the Dirac sea [24], are similar in nature and can be considered as spectral flows provided one identifies the effect of crossing over Schwarzschild horizon in the former with crossing over the zero energy level in the latter as both the cases involve a change in sign of energy. Clearly the horizon (being the crossover region) and energies close to the black hole singularity play the zero and $\Lambda$ energy levels respectively. This is also consistent with the accepted boundary condition of zero energy-momentum tensor at the horizon [2, 3]. Clearly this indicates that we are working in the Unruh [27] or Hartle-Hawking [28] vacuum. This is further consolidated by the fact, as we demonstrate later, that in our approach we recover the covariant anomaly. The connection between covariant boundary condition leading to covariant anomaly and the 
two above mentioned vacua are shown in [29]. Hence specification of the vacuum is inherent in our scheme of understanding the Hawking radiation.

In this note we will demonstrate that a proper marriage of the above two formalisms [2, 3] leads to the correct form gravitational anomaly structure. The details regarding computation of the correct numerical factor can be obtained from the work of Fumita [23]. To achieve this we will apply the formalism of deriving gravitational anomaly [24] in an external black hole background. In comparison with the case of chiral gauge anomaly [17, 18, 24], we will replace the spectral motion across zero energy level [17, 18, 24] by the crossing of the Schwarzschild horizon having a small width [3].

Before proceeding we mention a recent work [30] where it is argued that the event horizon separates the essentially quantum regime inside the horizon from the exterior classical region. In [31] also it is advocated that the superluminal states in the Kerr-Neumann disc can be considered as a Dirac sea of quantized negative energy states. In fact much earlier Chandrasekhar had speculated [32] on the significance of negative energy states in the context of super-radiance in Dirac waves in A Kerr-Neumann background.

We now cast the dynamics of a fermion in a curved spacetime [33] in an analogous form of the fermion in gauge interaction [17, 18, 23, 24] that is suitable to study the spectral flow. We consider the following metric,

$$
d s^{2}=g_{\mu \nu} d x^{\mu} d x^{\nu}=f(r) d t^{2}-f(r)^{-1} d r^{2}-r^{2} d \Omega_{(d-2)}^{2}
$$

in $1+1$-dimensions since near horizon the theory effectively becomes $1+1$-dimensional [43, 44, 3]. Next we define the zweibein $e_{\mu}^{a}$

$$
g_{\mu \nu}=\eta_{a b} e_{\mu}^{a} e_{\nu}^{b}, \quad \eta_{a b}=\text { diagonal }\{1,-1\}
$$

The flat Minkowski-space massless Dirac equation

$$
i \gamma^{a} \partial_{a} \psi=0, \quad\left\{\gamma^{a}, \gamma^{b}\right\}=2 \eta^{a b}
$$


in the curved background is generalized to [33],

$$
i \gamma^{\mu} \nabla_{\mu} \psi=0, \quad\left\{\gamma^{\mu}, \gamma^{\nu}\right\}=2 g^{\mu \nu}, \quad \nabla_{\mu}=\partial_{\mu}+\frac{1}{2} \sigma^{a b} \omega_{a b ; \mu}
$$

In (44) we have defined [33] $\sigma^{a b}=\frac{1}{4}\left[\gamma^{a}, \gamma^{b}\right], \gamma^{\mu}=\gamma^{a} E_{a}^{\mu}$ with $E_{a}^{\mu}$ being the inverse of $e_{\mu}^{a}$. For the metric in question (11) we have

$$
e_{\mu}^{a}=\left(\begin{array}{cc}
\sqrt{f} & 0 \\
0 & \frac{1}{\sqrt{f}}
\end{array}\right) \quad ; \quad E_{a}^{\mu}=\left(\begin{array}{cc}
\frac{1}{\sqrt{f}} & 0 \\
0 & \sqrt{f} .
\end{array}\right) .
$$

This brings the Dirac equation (44) to the following form,

$$
\left(\begin{array}{cc}
0 & i\left(\frac{\partial_{t}}{\sqrt{f}}+\frac{f^{\prime}}{4 \sqrt{f}}+\sqrt{f} \partial_{r}\right) \\
i\left(\frac{\partial_{t}}{\sqrt{f}}-\frac{f^{\prime}}{4 \sqrt{f}}-\sqrt{f} \partial_{r}\right) & 0
\end{array}\right) \psi=0 .
$$

To separate the chiral components we have used the convention [24]),

$$
\gamma^{0}=\sigma^{1} ; \quad \gamma^{1}=i \sigma^{2} ; \quad \gamma_{5}=i \gamma^{0} \gamma^{1}=-i \sigma^{3}
$$

We define the positive and negative chiral components $\psi_{ \pm}=\frac{1}{2}\left(1 \pm i \gamma_{5}\right) \psi$ and find

$$
\psi_{+}=\left(\begin{array}{c}
e^{i(-E t+p r)} \\
0
\end{array}\right) ; \psi_{-}=\left(\begin{array}{c}
0 \\
e^{i(-E t+p r)}
\end{array}\right)
$$

This yields the following set of algebraic equations,

$$
E_{-}=-\left(f p-\frac{1}{4 r_{h}}\right)-\frac{f^{\prime}}{4} ; \quad E_{+}=\left(f p-\frac{1}{4 r_{h}}\right)+\frac{f^{\prime}}{4}
$$

for $\psi_{-}$and $\psi_{+}$respectively and $f^{\prime}$ denotes $\partial_{r} f$. In the above we have taken the Schwarzschild black hole with $f=1-\left(r_{h}\right) / r$. Strictly speaking $E_{ \pm}$are the energy levels for constant potential but we need to generalize them for adiabatically varying situation. But before elaborating on this let us discuss in some detail the already well known derivation of chiral gauge anomaly in a structurally similar formalism. This is the central idea in our scheme.

The flat space Dirac equation for massless electrodynamics is

$$
\gamma^{a}\left(i \partial_{a}-e A_{a}\right) \psi=0
$$


and this reduces to [24]

$$
E_{-}=-p+e A_{1} \quad ; \quad E_{+}=p-e A_{1}
$$

for $\psi_{+}$and $\psi_{-}$respectively and $\dot{A}_{1}=-E$, the electric field. (The analogous set of equations in our case are (44) and (8) respectively. Before proceeding further I should mention how to interpret (10) and (8) . The notation $E_{ \pm}$are not to be understood as energy eigenvalues. In fact they would have been energy levels for constant potentials. These relations are in a classical setting and they simply show in a heuristic way how a change of potential with time induces the spectral flow. A detailed derivation of the anomaly equation exploiting classical physics is provided in [34] where the underlying quantum nature of the field theory manifests itself only through the negative energy Dirac sea. In fact the true energy levels are not at all needed for the present derivation (see for example [17, 18, 21, 24, 23]).

More specifically, in $A_{0}=0$ gauge, the only non-zero gauge invariant quantity is the electric field $E=\partial_{t} A_{1}$ and this time variation is introduced in $A_{1}$ (for the chiral gauge anomaly case in [24] by Jackiw) as an adiabatic change $A_{1} \rightarrow A_{1}+\delta A_{1}$. For an adiabatic change the structure of the energy spectrum is not changed but only the explicit value of the energy level gets altered. Hence the particle states are quasi-stationary in nature.

A change in the gauge field induces the spectral flow across the zero energy level which signals the creation of one type of chirality. This is directly identified as the rate of change of fermion number of that chirality. This can be visualized from the Figures 1 and 2 [24]. In Figures 1 and 2 we plot the dispersions (10) for $A_{1}=0$ and for a non-zero $A_{1}$ with $\delta A_{1}>0$ respectively. The acute and obtuse angled branches refer to left handed and right handed chirality states respectively. The filled states are shaded circles whereas the empty states are clear circles. The ground state for $A_{1}=0$ is shown in Figure 1 with the filled up negative energy sea. In Figure 2 the movement of the states (or the spectral flow) across the zero energy level for an adiabatic change in $A_{1}$ with $\delta A_{1}>0$ is shown by the arrows and both branches contribute equally to the creation of right handed chirality. For a quantization length $L$ with density of states per length $L$ being $L / 2 \pi$, the rate of production of $\mathrm{RH}$ 
particle is

$$
\dot{N}_{R H}=L^{-1}(L / 2 \pi) \dot{\omega}_{F S}=(e / 2 \pi) E,
$$

where $\omega_{F S}$ is the energy at the Fermi surface. Using the massless dispersion relation for energy $\omega$ and momentum $P$ and Newtonian dynamics one connects $\dot{\omega}=\dot{P}=e E$. To this one adds the identical contribution coming from the Left Handed sector and thus obtains the axial anomaly as $\dot{N}_{R H}+\dot{N}_{L H}=(e / \pi) E$. This is the standard "Quantum Mechanics" derivation [17, 18, 24] where only the effect at the zero energy level appears.

In the field theoretic analysis of Fumita [23] one studies the classical conservation law

$$
\partial_{\mu} j_{5[-\epsilon(\Pi)]}^{\mu}=0 ; \quad j_{5}^{\mu}=\bar{\psi} \gamma^{\mu} \gamma_{5} \psi
$$

where $j_{5 \omega(\Pi)}^{\mu}$ denotes the axial current for energy $\omega=\Pi=P-e A_{1}$. For the anomaly one takes into account the whole Dirac sea by integrating the regularized current:

$$
L \int \frac{d \Pi}{2 \pi} j_{5[-\epsilon(\Pi)]}^{\mu} \exp \left(-\frac{\Pi^{2}}{\Lambda^{2}}\right) .
$$

Using the spectral flow arguments as before for the energy level $\Lambda$ one recovers [23] the anomalous Ward identity

$$
\partial_{\mu} j_{5}^{\mu}=\frac{e E}{\pi}
$$

As we pointed out before, in actuality $j_{5}^{\mu}$ is the fermion number current and does not contain any $\Pi$ and thus one can get the correct answer without considering the whole Dirac sea or a regularized current [17, 18].

Now we come to the gravitational anomaly. In Figure 3 we have tried a similar picturization (as the gauge anomaly explained previously) corresponding to (8). The first and second diagrams in Figure 3 depict the spectra for the particle (8) outside and inside the horizon respectively. Upper halfs of both the diagrams are only relevant that show a preferential change in the chirality content after crossing inside the horizon. Clearly $f^{\prime}$ in (8) plays the role of the gauge field. If we apply our mechanism in a thin slab straddling the horizon [3] the change in the gravitational field witnessed by a particle as it crosses the horizon will be 
$\epsilon f^{\prime \prime}$ with $\epsilon$ being the thickness of the slab. Taking a time derivative yields

$$
\epsilon \dot{f}^{\prime \prime}=\epsilon f^{\prime \prime \prime} \dot{r}=\epsilon f^{\prime \prime \prime} f
$$

In the last step we have considered the radial null geodesics and have used $\dot{r}=f$. Considering the above equation (15) per unit length, one gets the correct structure of the covariant form of gravitational anomaly [35]-[39], [3] as $f^{\prime \prime \prime} f$. Conceptually, the most direct usage of black hole physics in the present paper lies in the part of exploiting the radial null geodesic condition in replacing $\dot{r}$ by $f$. We are still not finished since the correct numerical factor in the anomaly has to be ascertained. In fact, in analogy with the fermion case, what we have obtained is the anomaly in the fermion number and not the gravitational anomaly that deals with the energy-momentum current (schematically $\sim \bar{\psi} \gamma^{\mu} \partial^{\nu} \gamma_{5} \psi \sim k^{\nu} \bar{\psi} \gamma^{\mu} \gamma_{5} \psi$ ). This requires a careful analysis since one has to consider the energy contribution due to the above for each negative energy level and then integrate over the whole Dirac sea with proper regularization. However, it is important to note that the above analytic expression $f^{\prime \prime \prime} f$ for each level is independent of the level momentum (or energy) and will come out of the energy integral of the Dirac sea which will finally reproduce only the numerical factor. But precisely this effect has already been computed by Fumita [23] and we simply borrow his result. Fumita in [23] discusses both the boson and fermion sectors in conformal gauge from the spectral flow point of view. For the fermions, the regularized vacuum energy functional [23]

$$
E[\phi(x)]=-L \int_{0}^{\Lambda} \frac{d k}{2 \pi} \frac{|k|}{2}<\psi_{k}, \exp \left(-\frac{\Delta^{(1 / 2)}}{\Lambda^{2}} \psi_{k}>_{\text {spinor }},\right.
$$

has to be calculated where the positive energy wavefunction of momentum $k$ is $\psi_{k}=$ $\exp (i k x-i|k| t) / \sqrt{L}$ which is right (left) handed for $k>0(k<0)$. For Majorana fermions with conformal weight $1 / 2$ the general expression for the one dimensional Laplacian $\Delta=-\left|g^{11}(x)\right| \nabla_{1} \nabla_{1}$ where

$$
\Delta^{(j)}=-e^{-\phi}\left(\partial_{1}-\frac{j+1}{2} \partial_{1} \phi\right)\left(\partial_{1}-\frac{j}{2} \partial_{1} \phi\right)
$$

reduces to $\Delta^{(1 / 2)}$. Also the limits of integration relates to the horizon and the bottom of the Dirac sea, as explained earlier. The Liouville action is correctly reproduced (for details see 
[23]),

$$
E[\phi(x)]=\int d x\left[-\frac{\Lambda^{2}}{4 \pi} e^{\phi}-\frac{1 / 2}{96 \pi}\left(\partial_{1} \phi\right)^{2}+\frac{1}{48 \pi} \partial_{1}^{2} \phi+O\left(\frac{1}{\Lambda^{2}}\right)\right] .
$$

From this using the definition $T_{\mu \nu}=\frac{2}{\sqrt{-g}} \frac{\delta E[\phi]}{\delta g^{\mu \nu}}$ for the energy momentum tensor one recovers the correct form of conformal (or Weyl) anomaly,

$$
T_{\mu}^{\mu}=-\frac{f^{\prime \prime}}{48 \pi} .
$$

To bring the anomaly in this form, we had to use the parameterization $\exp (2 \phi)=f$ and subsequently use $\left(\partial / \partial_{x}=f\left(\partial / \partial_{r}\right)\right.$ to go to the Schwarzschild gauge (from the conformal gauge) [40, 41, 42]. From the trace anomaly itself Christensen and Fulling [43, 44] have shown how to derive the Hawking radiation. Hence, comparing with our previously obtained anomaly expression (15), we find that the correct numerical factor is $1 /(96 \pi)$ [35]-[39].

Finally, Hawking radiation can consist of both fermions as well as bosons. For the latter there is a consistent formulation [45, 46] with a Dirac like negative energy sea for the vacuum. Also in the paper by Fumita [23] (whose formalism we have adopted) both fermions and bosons are considered. Hence it is expected the same excercize can be repeated for the bosons as well.

To conclude, we have achieved what we set out to prove: the understanding of Hawking radiation as a tunnelling phenomenon [2] and from gravitational anomaly framework [3] are not to be considered as isolated and distinct formalisms. On the other hand, as we show here, crossing the horizon (or the tunnelling picture) generates the anomaly when one views it as a spectral flow of energy levels from one signature to another as it crosses the zero level or horizon. We stress that in our derivation we have used boundary conditions that are consistent with the accepted one that the energy momentum flux on the horizon is zero (the Unruh or Hartle-Hawking vacua). The fact that we are considering black hole physics (instead of the conventional anomaly as in [23]) comes out strongly in our use of the null radial null geodesics condition on the horizon (in the derivation of (15)). Lastly we point out that the numerical factor for the anomaly also comes correctly (using the analysis of [23]) 
thereby demonstrating consistency and completeness of the spectral flow framework.

Acknowledgements: Discussions with Rabin Banerjee, Bibhas Ranjan Majhi and Sujoy Modak are gratefully acknowledged. Also I thank Satya Prakash Ojha for his help in drawing the figures.

\section{References}

[1] S.Hawking, Commun.Math.Phys. 43 (1975) 199.

[2] M.K.Parikh and F.Wilczek, Phys.Rev.Lett. 85 (2000) 5042 arxiv:hep-th/9907001.

[3] S.P.Robinson and F.Wilczek, Phys.Rev.Lett. 95 (2005) 011303 arxiv:gr-qc/0502074.

[4] S.Iso, H.Umetsu and F.Wilczek, Phys.Rev.Lett. $96 \quad$ (2006) 151302 arxiv:hep-th/0602146.

[5] K.Murata and J.Soda, Phys.Rev. D74 (2006)044018 arxiv:hep-th/0606069.

[6] E.Vagenas and S.Das, JHEP 0704 (2007) 068 [arxiv:hep-th/0606077.

[7] M.R.Setare, Eur.Phys.J. C49 (2007) 865 arxiv:hep-th/0608080].

[8] Q.Q.Jiang and S.Q.Wu, Phys.Lett. B647 (2007)200 arxiv:hep-th/0701002.

[9] H.Shin and W.Kim, arXiv:0705.0265.

[10] S.Das, S.P.Robinson and E.C.Vagenas, arXiv: 0705.2233.

[11] R.Banerjee and S.Kulkarni, Phys. Rev. D 77, 024018 (2008) [arXiv:0707.2449].

[12] R.Banerjee and B.R.Majhi, Phys.Lett.B662 62 (2008) arXiv:0801.0200].

[13] L. Bonora, M. Cvitan, JHEP05(2008)071 (arXiv:0804.0198).

[14] L.Bonora, M.Cvitan, S.Pallua and I.Smolic, arXiv:0808.2360. 
[15] M.K.Parikh, Int.J.Mod.Phys. D13 (2004) 2351 [hep-th/0405160].

[16] M.K.Parikh, hep-th/0402166.

[17] H.B.Nielsen and M.Ninomiya, Nucl.Phys. B185 (1981)20.

[18] H.B.Nielsen and M.Ninomiya, Phys.Lett. 105B (1981) 219.

[19] G.'Hooft, Phys.Rev.Lett. 37 (1976) 8.

[20] A.S.Blaer, N.H.Christ and J.-F. Tang, Phys.Rev.Lett. 47 (1981) 1364.

[21] H.B.Nielsen and M.Ninomiya, Phys.Lett. 130B (1983)389.

[22] H.B.Nielsen, Talk at Trieste Conference on "Topological Methods in Quantum Field Theory", Trieste, 1990.

[23] N.Fumita, Int.J.Mod.Phys. A10 (1995) 2579 arxiv:hep-th/9406037.

[24] R.Jackiw, Dirac Prize Lecture, Trieste 1999 arxiv:hep-th/9903255.

[25] S.Adler, Phys.Rev. 177 (1969) 2426.

[26] J.S.Bell and R.Jackiw, Nuovo Cimento 60A (1969) 4.

[27] W.G.Unruh, Phys.Rev.D14 (1976) 870.

[28] J.B.Hartle and S.W.Hawking, Phys.Rev. D13 (10976) 2188.

[29] R.Banerjee and S.Kulkarni, arXiv:0810.5683.

[30] B.P.Kosyakov, Found.Phys. 38 (2008)678.

[31] R.Bock, arXiv: 0806.1674.

[32] S.Chandrasekhar, The Mathematical Theory of Black Holes, Johm Wiley: New York (1984). 
[33] S.K.Moayedi and F.Darabi, Phys.Lett. A322 (2004) 173 arXiv:gr-qc/0208053].

[34] A Widom and Y.Srivastava, Am.J.Phys. 56 (9) (1988)824.

[35] L.Alvarez-Gaume and E.Witten, Nucl.Phys. B234 (1983) 269.

[36] W.A.Bardeen and B.Zumino, Nucl.Phys. B244 (1984) 421.

[37] K.Fujikawa, Z.Phys. C28 (1985) 289.

[38] R.A.Bertlmann and E.Kohlprath, Ann.Phys. 288 (2001) 137 arxiv:hep-th/0011067.

[39] See also R.A.Bertlmann, Anomalies in Quantum Field Theory, Oxford Sciences, Oxford, 2004.

[40] D.Grumiller, W.Kummer and D.V.Vassilevich, Phys.Rept. $369 \quad$ (2002) 327 [hep-th/0204253v9].

[41] D.R.Karakhanyan, R.P.Manvelyan and R.L.Mkrtchyan, Phys.Lett.B 329 (1994) 185.

[42] R.Jackiw, arXiv:hep-th/9501016.

[43] S.Christensen and S.Fulling, Phys.Rev. D15 (1977) 2088.

[44] R.Balbinot and A.Fabbri, Phys.Rev. D59 (1999) 044031 hep-th/9807123v1].

[45] Y.Habara, Y.Nagatani, H.B.Nielsen and M.Ninomiya, Int.J.Mod.Phys.A23 (2008) 2771 (arXiv:hep-th/0607182).

[46] Y.Habara, Y.Nagatani, H.B.Nielsen and M.Ninomiya, Int.J.Mod.Phys.A23 (2008) 2733 (arXiv:hep-th/0603242). 


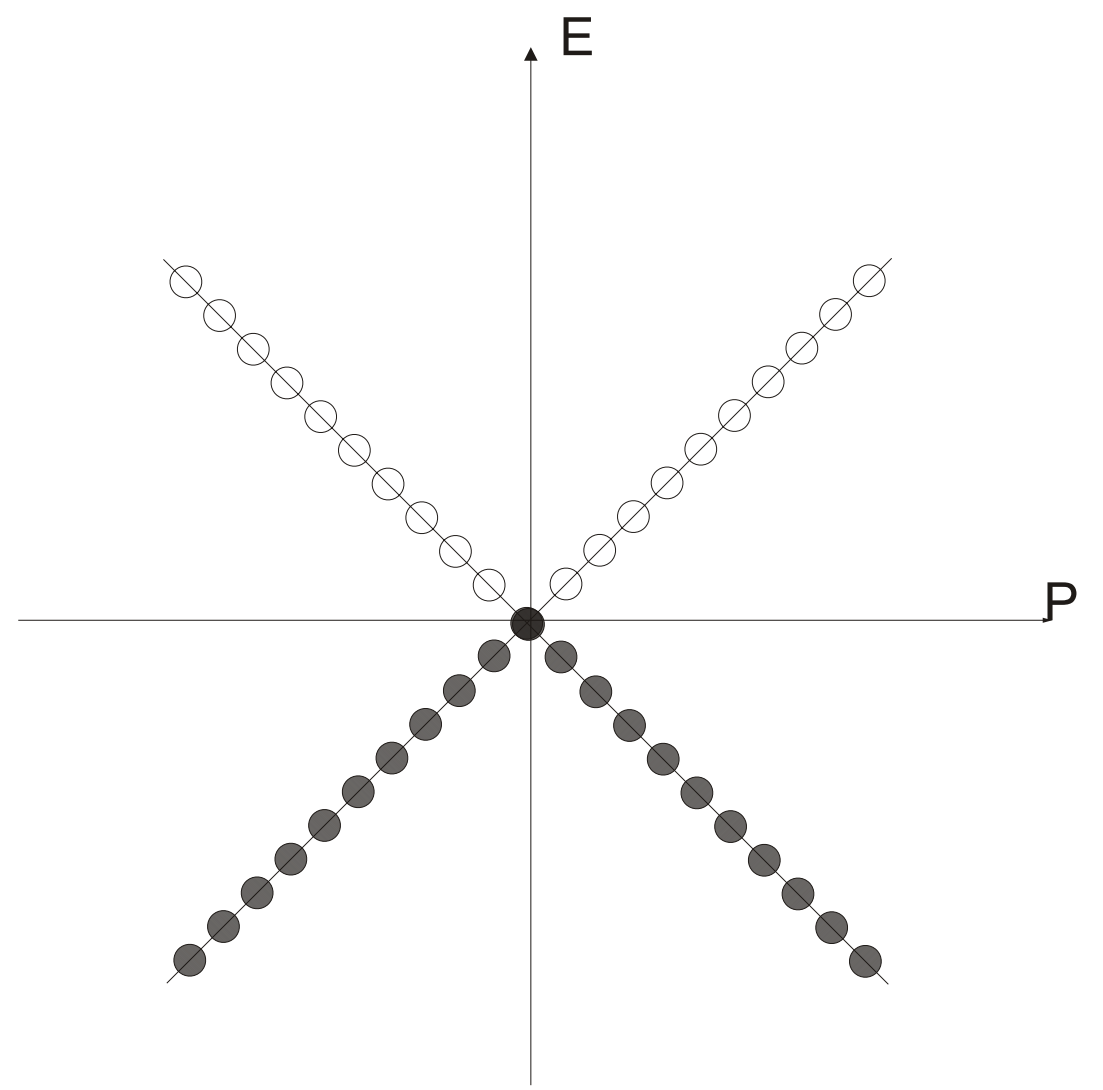

Figure 1: Ground state spectrum for $A=0$ 


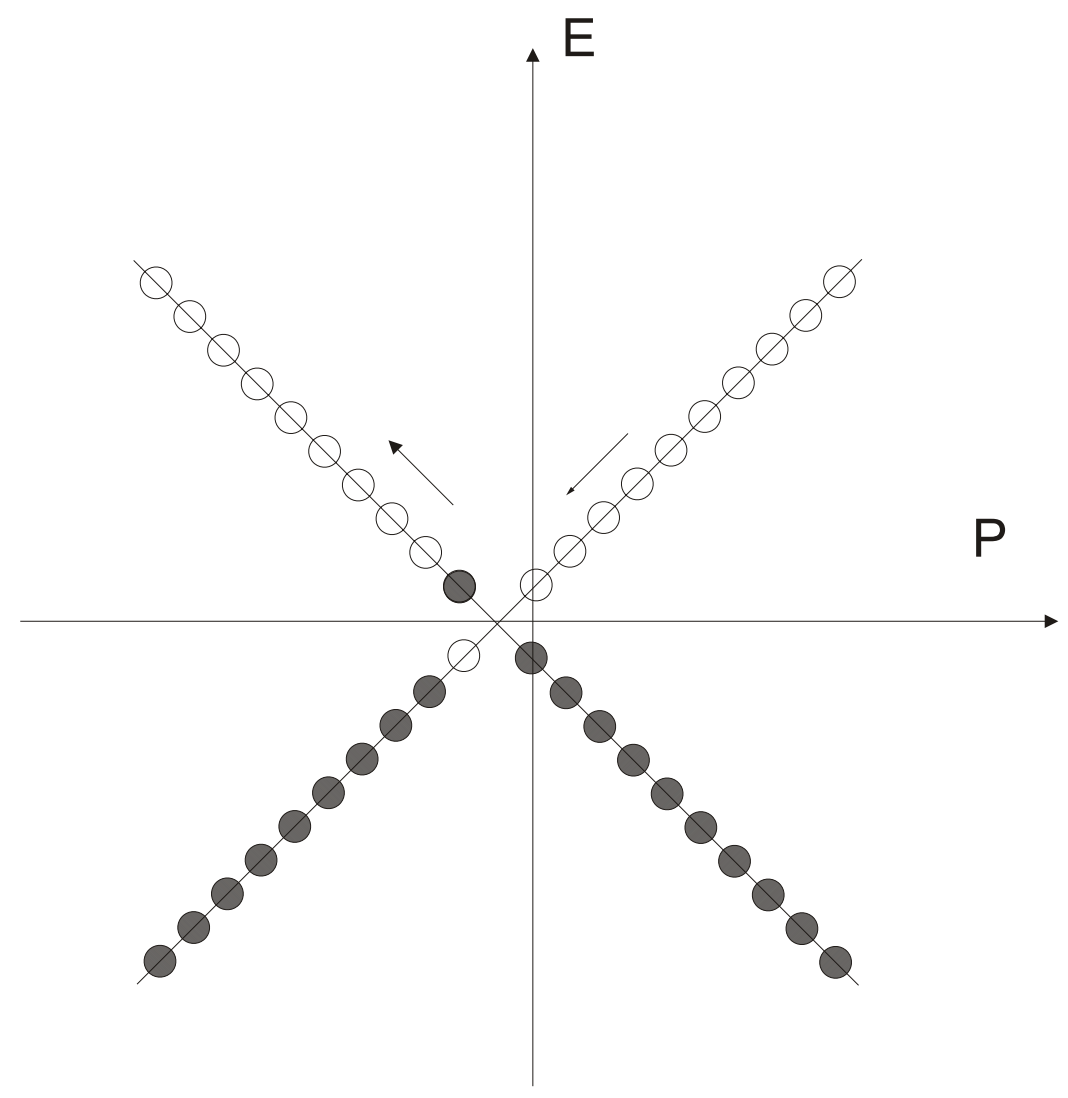

Figure 2: Spectrum for nonzero A 


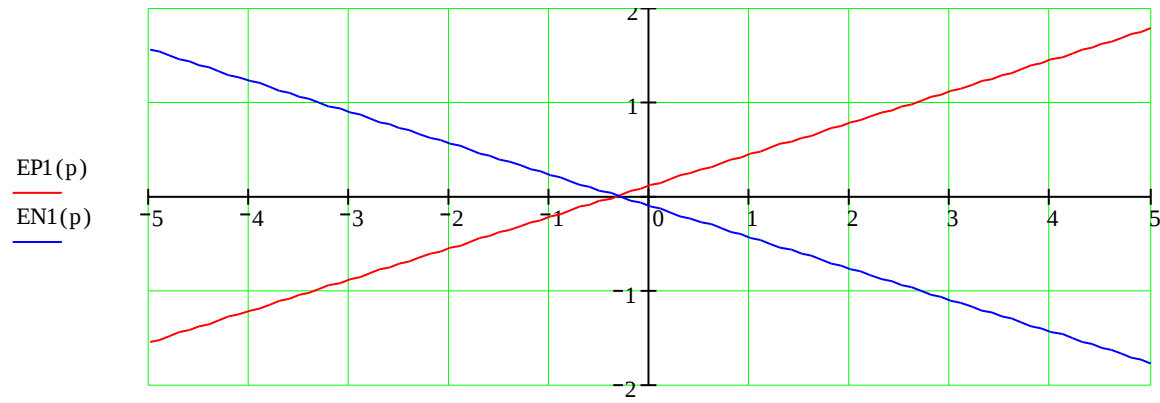

p

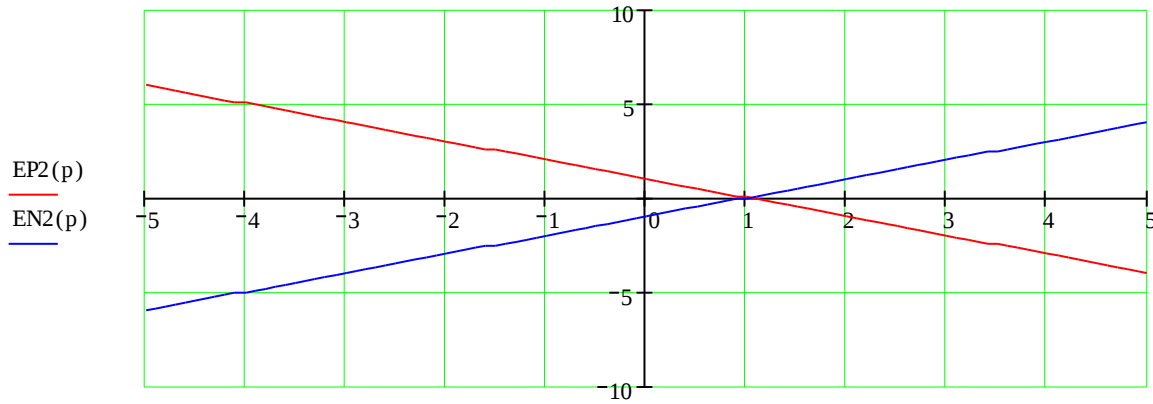

$\mathrm{p}$

Figure 3: EP1(p) and EN1(p) are the positive and negative chiralities for a fixed $r=1.5$. Similarly EP2(p) and EN2(p) are chiralities for a fixed $r=0.5$. The black hole horizon is at $r=1$. 